\title{
High oesophageal stricture with hiatal hernia and a lower oesophagus lined by columnar epithelium
}

\author{
B. CORRIN, G. KENT HARR ISON, H.R. M. JOH N S ON \\ St. Thomas's Hospital and Medical School, London, S.E.1
}

\begin{abstract}
A 23-year-old man with a six months' history of dysphagia was found to have hiatus hernia with reflux and a stricture of the mid-oesophagus. Death from unrelated causes permitted detailed histological examination. This showed a high squamo-columnar junction with ulceration confined to the squamous epithelium immediately above the junction. The columnar epithelium of the lower oesophagus was largely of cardiac type, but a few gastric body type glands were present at the lowermost end. This mixed pattern and the complete lack of inflammation or fibrosis beneath the columnar epithelium favours a congenital rather than metaplastic origin. A high squamocolumnar junction is of clinical significance only in the presence of reflux when oesophagitis and stricture develop at an unusually high level.
\end{abstract}

Since Barrett (1950) first drew attention to this condition several cases have been reported, many with histological detail provided by multiple biopsies (Adler, 1963; Abrams and Heath, 1965). These have generally shown squamous epithelium above the stricture and non-acid-secreting columnar epithelium below. It is unclear, however, whether it is the junctional area itself that is being attacked or one or other of these epithelia selectively. Another problem concerns the nature of the columnar epithelium in the oesophagus, whether it is a congenital anomaly or a reaction to inflammation. We have recently had the opportunity to study a case post mortem, when we were able to localize the area of ulceration accurately and to examine the deeper layers of the lower oesophagus for evidence of preceding inflammation, so obtaining information relevant to both these problems.

\section{CASE REPORT}

A 23-year-old man presented with dysphagia of six months' duration. The dysphagia had steadily increased to the point when fluids could not be swallowed. Pain was not significant at any time. No history of indigestion and no symptoms of gastric reflux could be obtained previous to six months.

Barium swallow showed almost complete obstruction in the mid-oesophagus. Oesophagoscopy showed a stricture about $20 \mathrm{~cm}$. from the superior alveolar margin. This was dilated and biopsies were taken. The Figure shows a barium meal after dilatation, with

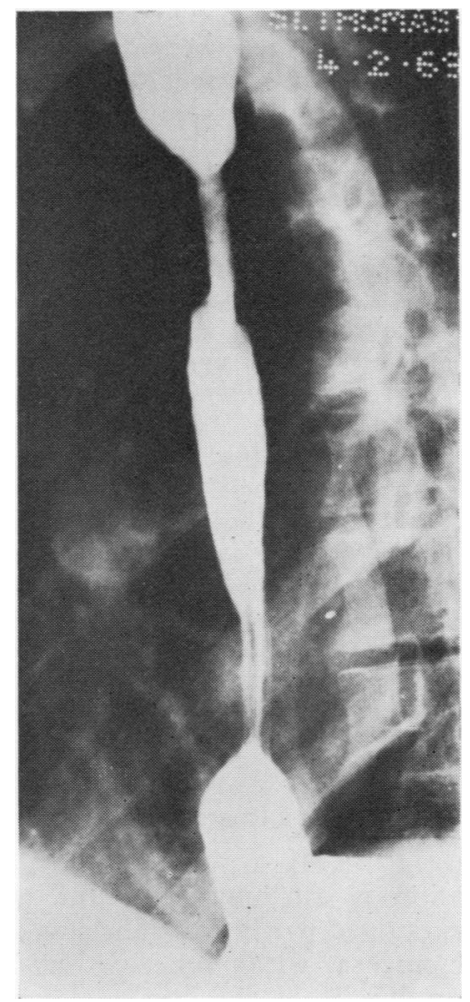

FIGURE. Barium meal showing hiatus hernia and stricture. The lower constriction is a peristaltic wave. 
the stricture and the hiatus hernia demonstrated. Moderate reflux was present. The stricture was 3.5 $\mathrm{cm}$. long and the lower end of the stricture was 11 $\mathrm{cm}$. above the stomach as measured on the radiograph.

Death occurred from complications following an operation which restored the abdominal oesophagus.

POST-MORTEM EXAMINATION Significant findings were confined to the oesophagus, stomach, and duodenum. Death was due to massive gastro-intestinal haemorrhage from multiple acute peptic ulcers of the stomach and duodenum. The oesophagus was constricted over a $3-\mathrm{cm}$. length at the level of the carina. There was shallow ulceration of this region. Above the stricture the oesophagus was lined by squamous epithelium, whilst below its lining resembled that of the stomach. There was fibrous thickening of the oesophageal wall in the region of the ulcer but none elsewhere. The distance from the squamo-columnar junction, as identified histologically, to the external cardiac incisura measured $7 \mathrm{~cm}$. in the contracted fixed specimen.

Histologically the oesophageal ulcer was bordered at both its upper and lower margins by squamous epithelium and the squamo-columnar junction was situated $0.5 \mathrm{~cm}$. below the lower border of the ulcer. The appearance of the epithelium lining the lower oesophagus was complex but consisted basically of a simple columnar-celled mucus-secreting epithelium with underlying glands lined by similar cells. The glands were limited to the mucosa and the true oeosophageal glands which are situated in the submucosa were not observed. This type of mucosa appeared identical to that normally found in the cardia of the stomach. At the lowermost end of the oesophagus the glands also contained oxyntic cells, so that here the mucosa was of gastric body type. In addition to these gastric types of mucosa there were intermingled many islands of small intestinal type mucosa with goblet cells and Paneth cells. This intestinal type of epithelium was best seen near the squamo-columnar junction and diminished downwards. The ulcer was shallow and its base consisted of chronic inflammatory granulation tissue and fibrous tissue. There was extensive fibrous replacement of the muscle coat beneath the ulcer but there was no inflammation or scarring whatsoever beneath the columnar epithelium of the lower oesophagus.

\section{DISCUSSION}

The ulceration is attributable to the action of peptic juice, derived partly from the small amount of fundic mucosa within the oesophagus but largely by reflux from the stomach, which was clearly demonstrated radiographically. The ulcera- tion began just above the squamo-columnar junc- $\frac{\overline{\bar{N}}}{9}$ tion, indicating that the columnar epithelium lining the lower part of the oesophagus was resistant to peptic digestion. Peptic digestion of $\frac{\omega}{\vec{D}}$ squamous epithelium results in shallow ulceration $\varrho$ which differs from the deep punched out ulcers os commonly seen in the stomach and duodenum $\overrightarrow{0}$ and infrequently encountered in a lower oesophagus lined by columnar epithelium (Barrett, $\vec{\omega}$ 1950 ; Adler, 1963).

The extensive columnar epithelium of the oeso- $\overrightarrow{\vec{x}}$ phagus could represent either a congenital o anomaly or metaplasia following reflux oeso- phagitis. The question has been discussed by Adler $\infty$ (1963), who favours the acquired theory. We are o influenced by the complete lack of inflammation $\underset{-}{-}$ or fibrous scarring beneath the columnar epi-c thelium and by the presence of a little fundic as well as cardiac type mucosa within the oeso- 을 phagus. Both these features we believe contraindicate a reactive change to previous oesophagitis $\vec{\theta}$ and therefore support a congenitally high squamocolumnar junction. The white line marking this junction is notoriously variable in its position, but in this case it appears to be exceptionally high. In the absence of reflux, extensive cardiac type mucosa in the oesophagus would be of no clinical $\frac{\circ}{D}$ significance. With reflux it results in the development of a stricture at an unusually high level. In our patient there was also a little fundic-type mucosa within the oesophagus, so that here peptic oesophagitis might have developed even in the absence of reflux.

Although we believe the gastric type mucosa is a congenital feature, the intestinal type mucosa probably represents a metaplastic change. Similar 3 appearances are found in the stomach accompanying gastritis, peptic ulceration, and carcinoma and $\frac{}{3}$ in the large bowel with ulcerative colitis. It is o significant that in the oesophagus the intestinal metaplasia was maximal nearest the ulcer.

In conclusion, we believe that this case demonstrates that, in the presence of gastric reflux, a $\widetilde{N}$ congenitally high squamo-columnar junction pro- $N$ tects the lower oesophagus and a stricture may develop at an unusually high level.

\section{REFERENCES}

Abrams, L., and Heath, D. (1965). Lower oesophagus lined with intestinal and gastric epithelia. Thorax, 20, 66

Adler, R. H. (1963). The lower esophagus lined by columnar epi- $T$ thelium. Its association with hiatal hernia, ulcer, stricture, and tumor. $J$. thorac. cardiovasc. Surg., 45, 13.

Barrett, N. R. (1950). Chronic peptic ulcer of the oesophagus and $\frac{\Omega}{\square}$ 'oesophagitis'. Brit. J. Surg., 38, 175. 